\title{
Extra cytoplasmic sigma factors in Staphylococcus aureus; their role and significance in the survival of Cocci
}

\begin{abstract}
Bacterial sigma $(\sigma)$ factors are an essential component of RNA polymerase. It determines the promoter selectivity. Alternative $\sigma$ factors play key roles in the coordinating gene transcription during various environmental stresses, nutrient starvation responses and during morphological development. The extra cytoplasmic function (ECF) $\sigma$ factors are small regulatory proteins that are quite divergent in sequence relative to most other $\sigma$ factors. In many bacteria with complex genome, contain multiple ECF $\sigma$ factors and these regulators often outnumber all other types of $\sigma$ factor combined. The roles and mechanisms of regulation for these various ECF $\sigma$ factors are largely unknown, but significant progress has been made in selected systems like Escherichia coli, Bacillus subtilis, Staphylococcus aureus, and Mycobacterium tuberculosis. Most ECF $\sigma$ factors are co-transcribed with one or more negative regulators. Upon receiving an appropriate environmental stimulus, the $\sigma$ factor is released and can bind to RNA polymerase to stimulate transcription. S. aureus causes a variety of human and animal infections. It produces multiple virulence factors as well as different regulators for controlling the expression of virulence factors. Prevention of staphylococcal infections is becoming problematic primarily because of the emergence and dissemination of the multiple-drug-resistant $S$. aureus strains across the world. Novel anti-staphylococcal drugs are required to eradicate all the $S$. aureus strains. The depth study of structural characterization of the primary and alternative sigma factors of $S$. aureus may provide a novel way for the development of anti-staphylococcal drug, as most of them are involved in the regulation of gene expression. The present review studies involve the important aspects ECF $\sigma$ factors in pathogenesis.
\end{abstract}

Volume I Issue 2 - 2016

\author{
Gajanan K Arbade \\ Department of Biosciences and Technology, Defence Institute of \\ Advanced Technology, India \\ Correspondence: Gajanan K Arbade, Department of \\ Biosciences and Technology, Defence Institute of Advanced \\ Technology, Girinagar, Pune-4I I025, MS, India, \\ Email gajananarbade@gmail.com
}

Received: October 25, 2016 | Published: December 07, 2016

Keywords: $\sigma$ factors, s. aureus, stress response, transcription regulation

\section{Abbreviations: ECF, extra cytoplas- mic function factors; ELE, enhancer-like elements;} RNAP, RNA polymerase; EBP, enhancer- binding proteins

\section{Introduction}

The initiation of transcription is the most important step of gene regulation in bacteria. For the initiation of transcription, RNA polymerase has to associate with a small protein, called sigma factor $(\sigma)$. This sigma factor helps to direct the RNA polymerase to bind to a specific class of Promoter sequence. Most bacterial species have ability to synthesize the different sigma- factors that recognize different promoter sequences. This is the reason why bacteria maintain basal gene expression as well as regulation of gene expression in response to altered environmental stimuli or developmental signals. ${ }^{1}$ Apart from these $\sigma$ factors bacteria encode thousands of different cell proteins necessary for the regular cellular activities and adaption to changed environmental conditions. The requirement of these protein is different as it changes with time and place in cell. Regulation of gene expression enables the cell to control the production of protein required to a cell at appropriate time of the cell cycle or for the adaption in the extracellular environmental changes. This regulation in turn makes it possible for the bacterium to adequately adapt to rapid changes in the environment. The regulation is either at transcription or at translation process. A key enzyme DNA-dependent RNA polymerase (RNAP) initiates the process of transcription which is the most prominent step in the gene regulation.
The DNA-dependent RNA polymerase (RANP) catalyzes the synthesis of RNA from a DNA template. However, RANP is not able to initiate the process of transcription by itself. In addition, it requires a polypeptide known as $\sigma$ factor. It is a family of relatively small molecular weight proteins most of the time associated with RANP in reversible manner. Collectively it forms an initiation specific enzyme, referred as holoenzyme. ${ }^{1}$

This variety in $\sigma$ factors provides the opportunity to the bacterium to maintain basal gene expression as well as for regulation of gene expression in response to altered environmental conditions. ${ }^{2}$ Bacterial initiation of transcription in is a multistep process which begins with the binding of RNAP holoenzyme, containing $\sigma 70$ or a $\sigma 70$-related $\sigma$ factor, to a specific DNA sequence recognized as a promoter. Recognition of a promoter sequence is directed primarily by the $\sigma$-subunit of the RNAP holoenzyme.

The binding of RNAP holoenzyme to the promoter results in formation of a closed complex, which is converted into an open complex by melting of a short region of DNA within the sequence bound by the enzyme. In the resulting ternary complex, the RNAP holoenzyme starts to produce small 2-12 base pairs RNA molecules, but remains located at the promoter. After dissociation of the $\sigma$ subunit, the RNAP core enzyme moves along the DNA, meanwhile synthesizing the nascent RNA molecule. A locally unwound region of DNA moves with the enzyme. The RNAP core enzyme and RNA are released when a terminator structure or factor is encountered and 
the DNA is then fully restored to duplex condition. One can define the strength of promoter as the frequency at which the holoenzyme initiates the transcription and is influenced by the promoter sequence and the conformational state of the DNA in promoter region. ${ }^{1,3}$

\section{Different types of sigma $(\sigma)$ factors}

There are two families of bacterial sigma-proteins viz. $\sigma 70$-family and sigma $\sigma 54$ family. The $\sigma 70$ family includes subgroups; i) Primary $\sigma$-factors ii) the nonessential primary-like $\sigma$-factors iii) Alternative $\sigma$-factors: a) Flagellar $\sigma$-factors b) Extra cytoplasmic function $\sigma$-factors c) Heat shock $\sigma$-factors and iv) $\sigma$-factors involved in sporulation. The primary $\sigma$ factors are present in all known eubacteria. These primary $\sigma$ factors are responsible for the transcription of most genes expressed in exponentially growing cells and are essential for cell survival. Recent data suggest that there is only one primary $\sigma$ factor present in any given eubacterial species. ${ }^{3}$

\section{Sigma $70(\sigma 70)$ family protein}

Members of the $\sigma 70$ family of $\sigma$ factors are components of the RNA polymerase holoenzyme that direct bacterial or plastid core RNA polymerase to specific promoter elements that are situated -10 and -35 base-pairs upstream of transcription-initiation points. The members of this family also function as contact points for specific activator proteins for instance $\mathrm{PhoB}$ and perform a role in initiation of the process. The primary $\sigma$ factor, which is essential for general transcription in exponentially growing cells, is reversibly associated with RNA polymerase and can be replaced by alternative $\sigma$ factors that coordinately express genes involved in diverse functions, such as stress responses, morphological development and iron uptake. On the basis of gene structure and function, members of the $\sigma 70$ family can broadly be divided into four main groups.

Sequence alignment results of the family members divulge that they have four different conserved regions, even though among the four highest conservation is found in region 2 and region 4 . These two regions are involved in binding to RNA polymerase, recognizing promoters and more importantly melting of the DNA strands. The current structural data shows that, divisions of linear sequence of $\sigma 70 \sigma$ factors into four regions have three stable domains that contain region 2, 3 and region 4 . These domains are spread out across the face of RNA polymerase in holoenzyme. ${ }^{4}$ In Bacteria, the core RNA polymerase consists of five subunits $\left(\beta \beta^{\prime} \alpha 2 \omega\right)$, is sufficient for transcription elongation and termination but is unable to initiate transcription. Transcription initiation from promoter elements requires a sixth, dissociable subunit called an $\sigma$ factor, which reversibly associates with the core RNA polymerase complex to form a holoenzyme. The majority of $\sigma$ factors belong to the so-called $\sigma 70$ family, reflecting their relationship to the principal factor of $E$. coli, $\sigma 70$.

\section{Sigma $54(\sigma 54)$ family proteins}

A second family of factors, the $\sigma 54$ family, comprises proteins that are functionally similar to, but structurally distinct from, $\sigma 70$ of $E$. coli. $^{4}$ The $\sigma 54$ system is widely distributed in the alpha and gamma proteobacteria and it has also been reported to be present in B. subtilis.

Among other physiological processes controlled by $\sigma 54$ are nitrogen assimilation and fixation, dicarboxylic acid transport, hydrogen oxidation, alginate utilization, degradation of aromatic compounds, formation of Pilli and formate utilization. ${ }^{5}$ A common feature of all these processes is that they are not absolutely required for cell survival, and each is activated by a different EBP (enhancer binding protein) interacting with a distinctive ELE (enhancer like elements) nucleotide sequence. In addition to its ability to be activated at a distance, the E. coli $\sigma 54$ system has several other unique characteristics that are not common among bacterial holoenzymes. The $\sigma 54$ recognizes promoter DNA with unusually close boxes, centered at around -12 and -24 from the transcription start, instead of the more common -10 and -35 boxes Upon binding, E. coli $\sigma 54$ forms a stable, closed promoter complex, but isomerization to the transcriptionally active open promoter complex is absolutely dependent on an activator protein bound at the ELE. This is an energy requiring step and the energy is obtained from hydrolysis of ATP catalyzed by the EBP itself.

In this sense, $\sigma 54$ can be regarded as a defective holozyme that only initiates transcription in concert with a second transcriptional factor. The unique properties of $\sigma 54$ are also reflected in its structure. This protein is the only $\sigma$ factor that does not have sequence similarity to any other known prokaryotic $\sigma$ factor, indicating that $\sigma 54$ has a different origin than the rest of the known $\sigma$ factors. More importantly, it was found that several functional motifs normally found in eukaryotic transcriptional factors, such as a Leucine zipper, an acidic region, and a glutamine-rich region, have been identified in $\sigma 54 .{ }^{6}$ Another remarkable particularity of $\sigma 54$ is its ability to interact with promoter DNA in the absence of core RNA polymerase.

The distinctive form in which $\sigma 54$ interacts with the promoter DNA, the strict dependency on regulatory proteins bound far upstream, and the requirement for nucleotide hydrolysis for activation of transcription is reminiscent of the activation process by eukaryotic RNA polymerase II. ${ }^{7}$

Extra cytoplasmic function $\sigma$ factor Members of the extra cytoplasmic function subfamily of $\sigma 70 \sigma$ factors that regulates the functions related to sensing and responding to changes in the bacterial periplasm and extracellular environment. These $\sigma$ factors are conserved in both gram-positive and gram-negative bacteria species. The first ECF $\sigma$ factor identified was E. coli $\sigma \mathrm{E}$, which was recognized as a second heat shock $\sigma$ factor in this organism. Although $\sigma \mathrm{E}$ does not appear to effect virulence in E. coli, other ECF $\sigma$ factors contribute to regulation of virulence genes and virulence-associated genes in a number of bacteria, including $S$. aureus, S. enterica, S. typhimurium, P. aeruginosa, and $M$. tuberculosis.

ECF $\sigma$ subgroup of the $\sigma 70$-family is a class of environmentally responsive transcriptional regulator. ${ }^{8}$ The amino acid sequences of these $\sigma$ factors were so different from the $\sigma 70$-factors that they were initially not recognized as $\sigma$ factors. They share similarity with the primary $\sigma$ factors in three of the four conserved regions. Several bacterial species contain different members of ECF $\sigma$ factor family, controlling the variety of functions in accordance with the extracellular signals.

ECF $\sigma$ factors include $\mathrm{Alg} \mathrm{U}$, which regulates biosynthesis of alginate in Pseudomonas aeruginosa. Alginate is a viscous exopolysaccharide believed to protect the bacterium from the adverse environment of the cystic fibrosis lung, PbrA of Pseudomonas fluorescens involved in iron uptake. The light inducible biosynthesis of carotenoid in Myxococcus xanthus is controlled by CarQ. Carotenoids are often found in non-photosynthetic bacteria as lightprotective agents. The $S$. coelicolor $\sigma \mathrm{E}$ promotes transcription of a gene encoding an extracellular agar degrading enzyme, agarase, while $\sigma \mathrm{E}$ of Streptomyces antibioticus is required for the production of the 
antibiotic actinomycin. HprL of Pseudomonas syringae controls transcription and secretion of a plant virulence factor and is inducible by plant extracts. $\mathrm{CnrH}$ plays a role in resistance to nickel and cobalt efflux in Alcaligenes eutrophus. The Mycobacterium smegmatis ECF $\sigma$-factor plays an important to withstand a variety of stresses. The expression of several outer membrane proteins in the deepsea bacterium Photobacterium sp. strain SS9 is regulated by $\sigma \mathrm{E}$ in response to growth under hydrostatic pressure. $B$. subtilis contains five ECF $\sigma$ factors viz. SigV, SigW, SigX, SigY and SigZ. SigX is required for survival at high temperatures but the function of the other ECF $\sigma$ factors is unknown. The second ECF $\sigma$ factor of E. coli, $\sigma \mathrm{E}$ is induced not only by heat or ethanol but also by disruption of protein folding in the periplasm. ${ }^{1}$ Alternative $\sigma$ factors regulate the gene expression in response to different extracellular changes.

One such class of $\sigma$ factors appears to controls different functions, including expression of heat-shock genes in $E$. coli, biosynthesis of alginates and carotenoids in P. aeruginosa \& Myxococcus xanthus, respectively, uptake of iron in E. coli and Pseudomonas spp., nickel and cobalt efflux in Alcaligenes europhus, plant pathogenicity in Pseudomonas syringae and synthesis of outer membrane proteins in Photobacterium sp. Strain SS9. Most of these activities deal with extra cytoplasmic functions, and such $\sigma$ factors have been designated as ECF $\sigma$ factors. They have also been characterized in Mycobacteria as well as Gram-positive bacteria such as Streptomyces coelicolor and B. subtilis and the archaea Sulpholobus acidocaldarius.

ECF $\sigma$ factors belong to a subfamily of the $\sigma 70$ class, based on their sequence conservation and function across bacterial species. The promoter consensus sequences recognized by the ECF $\sigma$ factors are also highly conserved. In most of the cases, the activity of these factors is modulated by a cognate inner membrane protein that has been shown, both in E. coli and in P. aeruginosa, to act as an anti- $\sigma$ activity.

This inner membrane protein is presumed to serve as a sensor and signalling molecule, allowing an adaptive response to specific environmental change presumably, an on-and-off switch of the anti- $\sigma$ activity leads to the release of the $\sigma$ factor and thereby to the coordinate transcription of the specific regulatory gene. ${ }^{9}$

\section{Sigma $(\sigma)$ factors in Staphylococcus aureus}

There are only four sigma factors have been identified in $S$. aureus to date. $\sigma \mathrm{A}$ the housekeeping $\sigma$ factor, which directs the transcription of the bulk cellular RNA, and three alternative $\sigma$ factors $\sigma \mathrm{B}, \sigma \mathrm{H}$ and the newly defined $\sigma \mathrm{S}$. The $S$. aureus $\sigma \mathrm{B}$ protein is closely related to the $\sigma \mathrm{B}$ protein of $B$. subtilis, and is mainly involved in stress response. The $S$. aureus $\sigma \mathrm{H}$ protein is a homolog of $B$. subtilis $\sigma \mathrm{H}$ involved in the regulation of sporulation-related genes. ${ }^{10} S$. aureus relies on only four $\sigma$ factors to direct the execution of its gene expression. In addition to a primary $\sigma$ factor, $\sigma \mathrm{A}, S$. aureus, as with the majority of organisms possesses a $\sigma \mathrm{B}$ alternative $\sigma$ factor, which controls the general stress response. A third factor, $\sigma \mathrm{H}$, has recently been reported, demonstrating homology to $\sigma \mathrm{H}$ from $B$. subtilis, and has been shown to regulate competence genes and the integration and excision of prophage. Recently it was demonstrated that the existence of a fourth $\sigma$ factor, $\sigma \mathrm{S}$, belonging to the ECF family.

Unlike many other organisms, which commonly possess multiple $\mathrm{ECF} \sigma$ factors, $\sigma \mathrm{S}$ is the only such element discovered in this organism thus far. $\sigma S$ is important in extended survival during starvation and by lysis with Detergents like Triton X-100. It is also highly inducible in response to a variety of stresses, including those that elicit DNA damage and cell wall perturbations. ${ }^{11}$ It was noticed that thermal stability of primary $\sigma$ factor of $B$. subtilis is nearly similar to that of $S$. aureus $\sigma \mathrm{A}$ Taken together; it can be speculated that the primary $\sigma$ subunits, which share extensive sequence identity with those of $B$. subtilis and $S$. aureus, might be unstable while in free form. ${ }^{12}$ The primary $\sigma$ factor $\sigma \mathrm{A}$ of $S$. aureus, essential for $S$. aureus growth, it is homologous with that of $B$. subtilis and $E$. coli, shown to react with the antibody raised against the $B$. Subtilis or E. coli-specific primary $\sigma$ subunit. It was also recognized that several $S$. aureus promoters that are homologous to the promoters transcribed by the primary $\sigma$ factors of $E$. coli and B. Subtilis. The alternative $\sigma$ factor of $S$. aureus that transcribes many virulence genes also exhibited notable homology with $\sigma$ A.

Despite of the apparent differences from other bacterial primary $\sigma$ factors. Very little things are known about the stability of any primary $\sigma$ subunit while in free form. With recombinant $S$. aureus $\sigma$ A Miller and others in 2012 have reported its physicochemical properties and also demonstrated that it is a thermodynamically unstable protein, which is stabilized by binding with core RNA polymerase. ${ }^{11}$ The chromosomally encoded plaC gene was predicted to encode the primary $\sigma$ factor in $S$. aureus based on the amino acid sequence identity of the plaC gene product with that of the $B$. subtilis vegetative $\sigma$ factor $\sigma$ A. Deletion of the plaC gene from the chromosome shows the lethal effect on $S$. aureus. ${ }^{13}$ sigB controls the biosynthesis of staphyloxanthin, the characteristic golden pigment of $S$. aureus which is required for oxidative stress resistance. ${ }^{14}$ The sig B also found to be involved in desiccation tolerance. ${ }^{15}$ It has been recently shown that $\sigma \mathrm{B}$ is a potent and effective regulator of a wide range of genes. Numerous virulence-associated loci have been shown to be regulated by this factor, including coa, sarA, fnbA, clfA, sspA, hla, and seb.

The $\sigma \mathrm{B}$ has been shown to be a pleiotropic regulator of virulence determinant production and to be required for pathogenesis. ${ }^{16}$ In vitro studies of transcription of $S$. aureus SigB have been shown to participate in the transcription of the sar locus, a key regulator of virulence gene expression. Moreover, the expressions of lipase and thermo nuclease, which play crucial roles in abscess formation, have been associated with SigB control. Thus, SigB seems to contribute directly and indirectly in the expression of $S$. aureus virulence genes.

Most $\sigma$ factors are themselves transcriptionally regulated. A class of other proteins also controls the activity of $\sigma$ factor by post-translational known as anti- $\sigma$ factors, these proteins plays an important role in controlling the activity of $\sigma$ factors in some extent. Anti- $\sigma$ factor proteins bind and sequester to a specific $\sigma$ factor, and block the initiation of transcription. ${ }^{17}$ A novel regulator $S$. aureus, an as yet uncharacterized ECF- $\sigma$ factor $\sigma S$ that appears to be an important component of the stress and pathogenic responses of this organism. Using biochemical approaches, it has been proved that $\sigma \mathrm{S}$ are able to associates with core-RNAP, and initiate transcription from its own coding region. Using a mutant strain Shaw and colleagues, 2006 determined that $\sigma \mathrm{S}$ is important for $S$. aureus survival during starvation, extended exposure to elevated growth temperatures, and Triton X-100 induced lysis. Co-culture studies reveal that the $\sigma \mathrm{S}$ mutant is significantly outcompeted by its parental strain, which is only exacerbated during prolonged growth or in the presence of stressor compounds. Interestingly, transcriptional analysis determined that under standard conditions, S. aureus SH1000 does not initiate expression of sigS. ${ }^{16}$ Sigma H $(\sigma \mathrm{H})$ : A member of double stranded 
DNA viruses, a template phage takes on a bacterial RNA polymerase with an essential $\sigma$ factor to initiate the sequence of reactions. Among the four $\sigma$ factors reported in $S$. aureus $\sigma \mathrm{H}$ helps the prophage to complete the some of the stages of its life cycle. The recognition site for $\sigma \mathrm{H}$ is upstream of the open reading frame (ORF) of int gene, which encodes the integrase. Experimentally it was proved that deletion of $\sigma \mathrm{H}$ resulted in the decreased phage intigrase mRNA level and increase of the excised form of prophase genome under normal growth conditions. The abilities of spontaneous lysis and lysogenization in $S$. aureus were also affected. These results indicate that $S$. aureus $\sigma \mathrm{H}$ modulates the transcription of phage integrase, stabilizes the lysogeny in the host cell and may further influence the prophage life cycle and correlative bacterial virulence. ${ }^{10}$ Regulatory pathways: In $S$. aureus SigB operon is comprised of four genes, these genes are homologous to $B$. subtilis rsbU, rsbV, rsbW, and sigB. As in $B$. subtilis, all the genes in the operon are expressed during exponential growth, presumably from the $\sigma \mathrm{A}$ dependent promoter. By invitro transcription studies it was confirmed that the internal $\mathrm{PB}$ promoter is $\sigma \mathrm{B}$ dependent. Transcriptional regulation of sigB operon in $S$. aureus is complex, it generates multiple tranblockeds viz. a bicistronic sigB-rsbW tranblocked and a sigB monocistronic tranblocked. In addition to an auto regulatory role for $S$. aureus $\sigma \mathrm{B}$ under conditions of environmental stress an rsbV-W-sigB tranblocked was induced by the exposure of cells to either ethanol $(4 \%)$ or a heat shock $\left(48^{\circ} \mathrm{C}\right) .{ }^{18}$ The cytoplasmic stress activates the $\sigma \mathrm{H}$ intern regulates the effects of $\sigma \mathrm{H}$ by expressing the sigH genes, starvation responses activates the $\sigma \mathrm{S}$, then the $\sigma \mathrm{S}$ binds to RNAP and then expresses the sigS genes, similarly the $\sigma \mathrm{A}$ and $\sigma \mathrm{B}$ are activated in response to environmental signals. ${ }^{19}$

\section{Discussion and conclusion}

Bacteria utilize alternative $\sigma$ factors to regulate a wide range of physiological processes, especially in pathogenic bacteria; alternative $\sigma$ factors often affect virulence. Virulence effects can be mediated either through direct virulence gene regulation or indirectly, by regulating genes that increase fitness and pathogenicity of the bacterium during transmission and infection. Alternative $\sigma$ factors frequently have roles in virulence by regulating virulence-associated genes that aid in a bacterium's survival during infection and stress conditions. Another alternative $\sigma$ factor role that contributes to environmental survival, and has virulence implications, is regulation of biofilm formation, e.g., by $\sigma \mathrm{B}$ in $S$. aureus and $S$. epidermidis. Functional roles for alternative $\sigma$ factors can be clearly defined and highly specific (e g. sporulation $\sigma$ factors). A most alternative $\sigma$ factor contributes to multiple, diverse functions in a cell. The $\sigma$ factors are conserved across most of the bacterial pathogens. A comparison of homologous sigma factor functions among different bacterial genera reveals that the roles of $\sigma$ factors vary greatly among bacterial species, even for closely related species.

A common mechanism of virulence regulation by alternative $\sigma$ factors involves coordinated networks of $\sigma$ factors along with other transcriptional regulators. Alternative $\sigma$ factors may regulate not only individual genes involved in virulence but also other $\sigma$ factors or transcriptional regulators that in turn regulate virulence genes and virulence-associated genes. Four different sigma factors are known to be there in $S$. aureus. Among these four each has its importance in the regulatory aspects. $\sigma \mathrm{A}$ is a housekeeping $\sigma$ factor directs the transcription of bulk cellular RNA which regulates the normal physiological processes. $\sigma \mathrm{B}$ is a regulator of virulence determinant production, ${ }^{20}$ also required for pathogenesis, biosynthesis of staphyloxanthin and involved in desiccation tolerance. $\sigma \mathrm{S}$ is required for the survival during starvation, extended exposure to elevated growth temperatures, and Triton X-100 induced lysis. Fourth $\sigma$ factor called $\sigma$ His plays an important role in modulating the prophage integration and excision, it also modulates the transcription of phage integrase, stabilizes the lysogeny in the host cell, influence the prophage life cycle and correlative bacterial virulence.

Table I Specific roles of different $\sigma$ factors in S. aureus

\begin{tabular}{|c|c|}
\hline $\begin{array}{l}\text { Sigma } \\
\text { factor }\end{array}$ & Role(s) \\
\hline$\sigma \mathrm{A}$ & Housekeeping sigma factor directs the transcription of bulk cellular RNA. \\
\hline$\sigma B$ & $\begin{array}{l}\text { Regulator of virulence determinant production, to be required for pathogenesis, biosynthesis of staphyloxanthin, involved in } \\
\text { desiccation tolerance. }\end{array}$ \\
\hline$\sigma S$ & Survival during starvation, extended exposure to elevated growth temperatures, and Triton X-100 induced lysis. \\
\hline$\sigma \mathrm{H}$ & $\begin{array}{l}\text { Modulates prophage integration and excision, modulates the transcription of phage integrase, stabilizes the lysogeny in the host } \\
\text { cell, influence the prophage life cycle and correlative bacterial virulence. }\end{array}$ \\
\hline
\end{tabular}

Table 2 Regulators of sigma factors in Staphylococcus aureus

\begin{tabular}{|c|c|c|c|}
\hline Sigma factor & Activator(s), regulator(s) & Organism & Reference \\
\hline$\sigma \mathrm{A}$ & Nutrition stress, Antibiotics & S. aureus & 12,19 \\
\hline$\sigma \mathrm{B}$ & $\begin{array}{l}\text { Nutrient starvation, energy stress stationary phase, high salt, heat, ethanol, low temperature, acid } \\
\mathrm{pH} \text {, nitrosative stress, cell wall-active agents. }\end{array}$ & S. aureus & 20 \\
\hline$\sigma S$ & Starvation, stresses that DNA damage and cell wall damage, oxidative stress & S. aureus & 16,20 \\
\hline$\sigma \mathrm{H}$ & Phage II infection & S. aureus & 10 \\
\hline
\end{tabular}




\section{Acknowledgements}

Author thanks to Dr. Sandeep K Srivastava for constant encouragement and support. GKA is also thankful to Defence Institute of Advanced Technology (DIAT), Pune, India for providing Research fellowship.

\section{Conflict of interest}

The author declares no conflict of interest.

\section{References}

1. Woesten M. Eubacterial sigma-factors. FEMS Microbiology Reviews 1998;22(3):127-150.

2. Harley CB, Reynolds RP. Analysis of E. coli promoter sequences. $\mathrm{Nu}$ cleic Acids Res. 1987;15(5):2343-2361.

3. Gruber TM, Bryant DA. Molecular systematic studies of eubacteria, using 670 -type sigma factors of group 1 and group 2. J Bacteriol. 1997;179(5):1734-1747.

4. Paget MSB, Helmann JD. The sigma 70 family of sigma factors. Genome Biology. 2003;4(1):203.

5. Moretfl E, Segoviat L. The sigma 54 bacterial enhancer-binding protein family: mechanism of action and phylogenetic relationship of their functional domains. J Bacteriol. 1993;175(19):6067-6074.

6. Morett E, Buck M. Invivo Studies on the interaction of RNA polymerase- $\sigma 54$ with the Klebsiella Pneumoniae and Rhizobium Meliloti nifH promoters the role of NifA in the formation of an open promoter complex. J JIol Hid. 1993;210(1):65-77.

7. Wang W, Carey M, Gralla JD. Polymerase II promoter activation: closed complex formation and ATP-driven start site opening. Science. 1991;255(5043):450-453.

8. Helmann JD. The extra cytoplasmic function (ECF) sigma factors. Adv Microb Physiol. 2002;46:47-110.

9. Missiakas D, Raina S. The extracytoplasmic function sigma factors: role and regulation. Molecular Microbiology. 1998;28(6):1059-1066.
10. Liang T, Xiaoqian W, Sun B. Alternative sigma factor sigma modulates prophase integration and excision in Staphylococcus aureus. PLoS Pathogens. 2010;6(5):e1000888.

11. Miller HK, Carroll RK, Burda WN, et al. The extra cytoplasmic function sigma factor $\sigma$ s protects against both intracellular and extra cytoplasmic stresses in Staphylococcus aureus. J Bacteriol. 2012;194(16):43424354.

12. Mondal R, Ganguly T, Chanda PK, et al. Stabilization of the primary sigma factor of Staphylococcus aureus by Core RNA Polymerase. BMB Reports. 2010;43(3):176-181.

13. Deora R, Mishra TK. Characterization of the Primary $\sigma$ Factor of Staphylococcus aureus. J Bacteriol. 1996;271(36):21828-21834.

14. Kullik I, Giachino P, Fuchs T. Deletion of the alternative sigma factor $\sigma \mathrm{B}$ in Staphylococcus aureus reveals its function as a global regulator of virulence genes. J Bacteriol. 1998;180(18):4814-4820.

15. Chaibenjawong P, Foster SJ. Desiccation tolerance in Staphylococcus aureus. Archives of Microbiology. 2011;193(2):125-135.

16. Shaw LN, Aish J, Davenport JE, et al. Investigations into sigma B-modulated regulatory pathways governing extracellular virulence determinant production in staphylococcus aureus. J Bacteriol. 2006;188(17):60706080 .

17. Miyazaki E, Chen JM, Ko C, et al. The Staphylococcus aureus rsbW (orf159) gene encodes an anti-sigma factor of SigB. J Bacteriol. 1999;181(9):2846-2851.

18. Kazmierczak MJ, Wiedmann M, Boor KJ. Alternative sigma factors and their roles in bacterial virulence. Microbiol Mol Biol R. 2005;69(4):527_ 543

19. Fang F C. Sigma cascades in prokaryotic regulatory networks. Proc Nat Acad Sci USA. 2005;102(14):4933-4934.

20. Keith P. Bacterial stress responses as determinants of antimicrobial resistance. J Antimicrob Chemother. 2012;67(9):2069-2089. 